\title{
Global patterns and trends in colorectal cancer incidence in young adults
}

\author{
Rebecca L Siegel (D) ,' Lindsey A Torre, ${ }^{1}$ Isabelle Soerjomataram, ${ }^{2}$ Richard B Hayes, ${ }^{3}$ \\ Freddie Bray, ${ }^{2}$ Thomas K Weber, $^{4,5}$ Ahmedin Jemal $^{1}$
}

\begin{abstract}
- Additional material is published online only. To view please visit the journal online (http://dx.doi.org/10.1136/ gutjnl-2019-319511).

${ }^{1}$ Intramural Research Department, American Cancer Society, Atlanta, Georgia, USA ${ }^{2}$ Section of Cancer Surveillance, International Agency for Research on Cancer, Lyon, France

${ }^{3}$ Department of Population Health, New York University School of Medicine, New York, New York, USA

${ }^{4}$ Department of Surgery, Donald and Barbara Zucker School of Medicine at Hofstra/Northwell, Hempstead, New York, USA ${ }^{5}$ Department of Surgical Oncology, Northwell Health Cancer Institute, Great Neck, New York, USA
\end{abstract}

\section{Correspondence to}

Ms Rebecca L Siegel, American Cancer Society, Atlanta, GA 30303, USA;

rebecca.siegel@cancer.org

Received 22 July 2019 Revised 16 August 2019 Accepted 21 August 2019 Published Online First 5 September 2019

\begin{abstract}
Objective Early-onset colorectal cancer (CRC) is increasing in the USA despite rapid declines in older ages. Similar patterns are reported in Australia and Canada, but a comprehensive global analysis of contemporary data is lacking.

Design We extracted long-term data from Cancer Incidence in Five Continents and supplemental sources to report on worldwide CRC incidence rates and trends by age ( $20-49$ years and $\geq 50$ years) through diagnosis year 2012 or beyond (Australia, Finland, New Zealand, Norway, Sweden, USA).
\end{abstract}

Results During 2008-2012, age-standardised CRC incidence rates in adults $<50$ ranged from 3.5 per $100000(95 \% \mathrm{Cl} 3.2$ to 3.9) in India (Chennai) to $12.9(95 \% \mathrm{Cl} 12.6$ to 13.3$)$ in Korea. During the most recent decade of available data, incidence in adults $<50$ was stable in 14 of 36 countries; declined in Austria, Italy and Lithuania; and increased in 19 countries, nine of which had stable or declining trends in older adults (Australia, Canada, Denmark, Germany, New Zealand, Slovenia, Sweden, UK and USA). In Cyprus, Netherlands and Norway, inclines in incidence in young adults were twice as rapid as those in older adults (eg, Norway average annual per cent change (AAPC), $1.9(95 \% \mathrm{Cl} 1.4$ to 2.5$)$ vs $0.5(95 \% \mathrm{Cl} 0.3$ to $0.7))$. Among most high-income countries with longterm data, the uptick in early-onset disease began in the mid-1990s. The steepest increases in young adults were in Korea (AAPC, $4.2(95 \% \mathrm{Cl} 3.4$ to 5.0)) and New Zealand (AAPC, $4.0(95 \% \mathrm{Cl} 2.1$ to 6.0$))$.

Conclusion CRC incidence increased exclusively in young adults in nine high-income countries spanning three continents, potentially signalling changes in early-life exposures that influence large bowel carcinogenesis.

\section{INTRODUCTION}

Colorectal cancer (CRC) is the third most commonly diagnosed cancer worldwide, with an estimated 1.8 million new cases in $2018 .^{1}$ Global patterns vary widely and are strongly linked to human development index level, reflecting the adoption of western lifestyles that accompany economic transition and elevate risk. In general, CRC incidence is rising in low-income and middle-income countries but beginning to stabilise or decline in high-income countries, especially those that have implemented screening. ${ }^{2}$ However, accumulating evidence from studies

\section{Significance of this study}

What is already known on this subject?

- Colorectal cancer (CRC) incidence rates in young adults (aged $<50$ years) are increasing in several countries, despite declining rates in older adults. The extent to which this pattern is occurring on a global scale is unknown.

What are the new findings?

- CRC incidence rates are uniquely increasing in young adults in nine high-income countries (Germany, USA, Australia, Canada, New Zealand, UK, Denmark, Slovenia and Sweden) across North America, Europe and Oceania where rates in older adults are stable or declining. Conversely, CRC declined in young adults in only three countries (Italy, Austria and Lithuania) compared with 11 countries in adults 50 and older.

How might it impact on clinical practice in the foreseeable future?

- Improving awareness of the marked increases in young-onset CRC incidence could facilitate more diligent assessment of cancer family history by primary care clinicians, as well as follow-up of symptoms in young individuals, many of whom are diagnosed at a late stage. These findings also highlight the need for research on early-life exposures in relation to colorectal carcinogenesis.

of cancer registry data indicates that favourable overall trends are masking an increase in young-onset CRC in the USA, ${ }^{3-6}$ Australia $^{78}$ and Canada. ${ }^{9}$ Although the absolute risk of CRC in adults younger than 50 years is low relative to older adults, disease trends in young age groups are a key indicator of recent changes in risk factor exposures and often foreshadow the future cancer burden. ${ }^{10}$ In addition to country-specific analyses, there are recent reports on early-onset CRC occurrence in seven high-income countries, ${ }^{11}$ in Europe $^{12}$ and in 11 'industrialised' countries (data through 2007). ${ }^{13}$ However, a comprehensive examination of contemporary trends on a global scale is lacking. We analysed high-quality longterm population-based data on CRC occurrence by age at diagnosis for 43 countries covering six continents. 


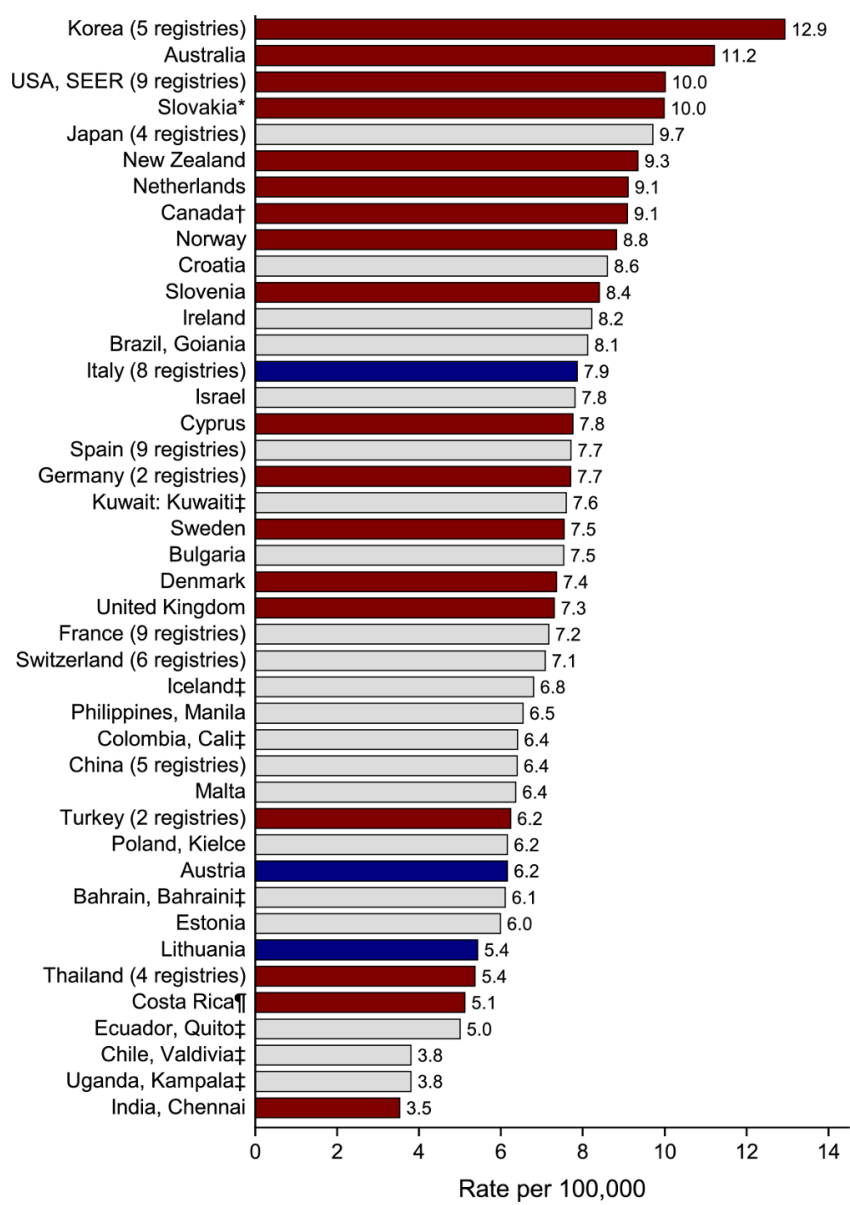

Figure 1 Age-standardised incidence rate during 2008-2012 for colorectal cancer among adults ages $20-49$ years. Bar shading indicates trend in incidence rates based on 10-year average annual per cent change; red: statistically significant increase; blue: statistically significant decrease; grey: stable or insufficient number of cases for trend analysis (¥). Rate for Finland unavailable. * Rate based on data during 2008-2010. tExcludes Nunavut, Quebec, and Yukon. EExcluded from trend analysis due to insufficient number of annual cases. ๆRate based on data during 2008-2011.

\section{METHODS}

We obtained high-quality population-based annual incidence data for colon and rectal cancer diagnosed through 2012 from the Cancer Incidence in Five Continents (CI5plus) database of the International Association of Cancer Registries and the International Agency for Research on Cancer (IARC). ${ }^{14}$ The CI5plus database is compiled from cancer registry data worldwide using a process that ensures comparable information that meets high quality standards established by IARC. Specifically, on submission the data coding is verified, the format is standardised, and an editorial board conducts an evaluation based on three dimensions of quality: comparability, completeness and validity. (For more information about the database, see ci5.iarc. fr/CI5-XI/Default.aspx.) The population coverage of registries included in CI5 may be national or subnational. If a country is represented by one or more registries but without national coverage, the registries are specified. National or subnational registries with cancer incidence data going back to at least 1998 were included and multiple datasets from subnational registries within a single country were combined, resulting in a total of 43 countries examined. To take advantage of the availability of more contemporary incidence, we obtained additional data by contacting individual registries or accessing publicly available data online. We acquired data through 2015 from Australia ( www.aihw.gov.au/); through 2016 from Finland (personal communication), New Zealand (personal communication) and the USA (seer.cancer.gov/data/); and through 2017 from Norway (personal communication) and Sweden (sdb.socialstyrelsen.se/ if_can/val.aspx). Seven countries had fewer than 10 CRC cases among ages 20-49 years in any single diagnosis year and were excluded from trend analysis.

Incidence was stratified by age at diagnosis, categorised as 20-49 years ('early-onset') or 50 years or older ('older adults'). Cancer subsite was categorised according to the International Classification of Diseases, 10th revision as colon (code C18) or rectum (code C19-C20). Given that trends in CRC incidence are quite similar in men and women overall ${ }^{2}$ and for earlyonset disease, ${ }^{6} 1516$ the two sexes were combined to improve stability. Primary outcome measures were average annual incidence rates during diagnosis years 2008-2012 (42 countries; data unavailable for Finland) and time-weighted average annual per cent change (AAPC) in incidence rates during the last 10 years of available data (36 countries) based on joinpoint regression analysis. This method fits joined straight lines (joinpoints) to observed annual age-standardised rates on a logarithmic scale. ${ }^{17}$ The maximum number of joinpoints is determined by the number of years available for each country/registry and was limited to four for countries with $\geq 24$ data years. Trends are described as 'increasing' or 'decreasing' if the AAPC is statistically significantly different from zero $(p<0.05)$ and 'stable' otherwise. All rates are expressed per 100000 population and age-standardised to the 1960 Segi world standard population (as modified by Doll and Cook). ${ }^{18}$ In a sensitivity analysis, we assessed the extent to which the inclusion of appendiceal cancer (C18.1) in the CI5plus grouping for colon cancer influenced our results by calculating rates and trends exclusive of appendix for three countries for which these data were available (USA, New Zealand and Canada).

\section{RESULTS}

\section{CRC incidence during 2008-2012}

Among 42 countries with high-quality population-based cancer registry data, cross-sectional age-standardised CRC incidence rates in ages 20-49 years during 2008-2012 were lowest in India (Chennai; 3.5 per 100000 (95\% CI 3.2 to 3.9)); Uganda (3.8, 95\% CI 3.0 to 4.6$)$; and Chile $(3.8,95 \%$ CI 2.5 to 5.1$)$ and highest in Korea $(12.9,95 \%$ CI 12.6 to 13.3); Australia (11.2, 95\% CI 10.9 to 11.5$)$; the USA $(10.0,95 \%$ CI 9.8 to $10.3)$; and Slovakia $(10.0,95 \%$ CI 9.3 to 10.7 ; figure 1 ; online supplementary table 1). The pattern in older adults was quite similar, with rates ranging from 27.5 (95\% CI 25.9 to 29.1) in India to 192.5 (95\% CI 188.6 to 196.3 ) in Slovakia (online supplementary table 2). Among young adults, incidence was generally higher for tumours developing in the colon than in the rectum, with a more than twofold difference in rates in Iceland, Italy and Cyprus (online supplementary tables 3 and 4). Exceptions were in Slovenia, where rates were similar, and Korea, India and China, where rates were slightly higher for rectal cancer. In contrast, incidence rates in older adults were a minimum of $7 \%$ higher for colon cancer than for rectal cancer (India, 14.2 per 100000 vs 13.3) and commonly twofold higher, particularly in high incidence countries (online supplementary tables 5 and 6). 
A

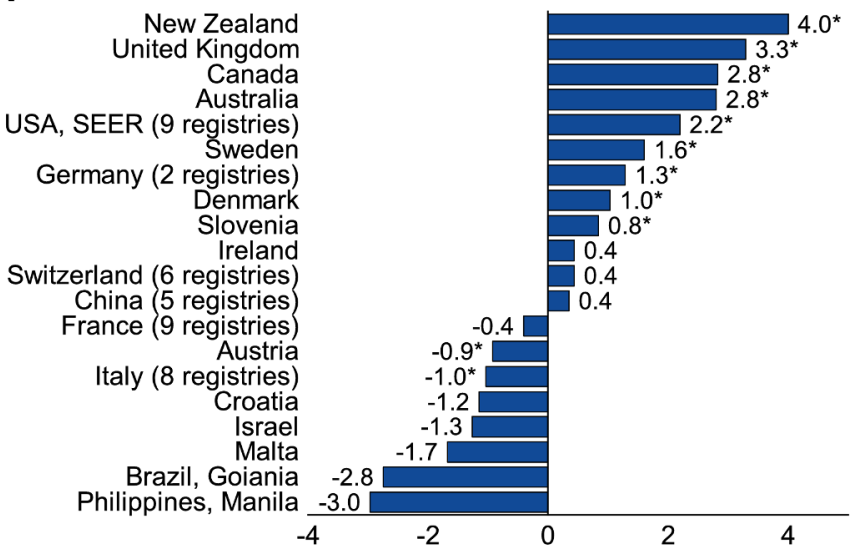

B

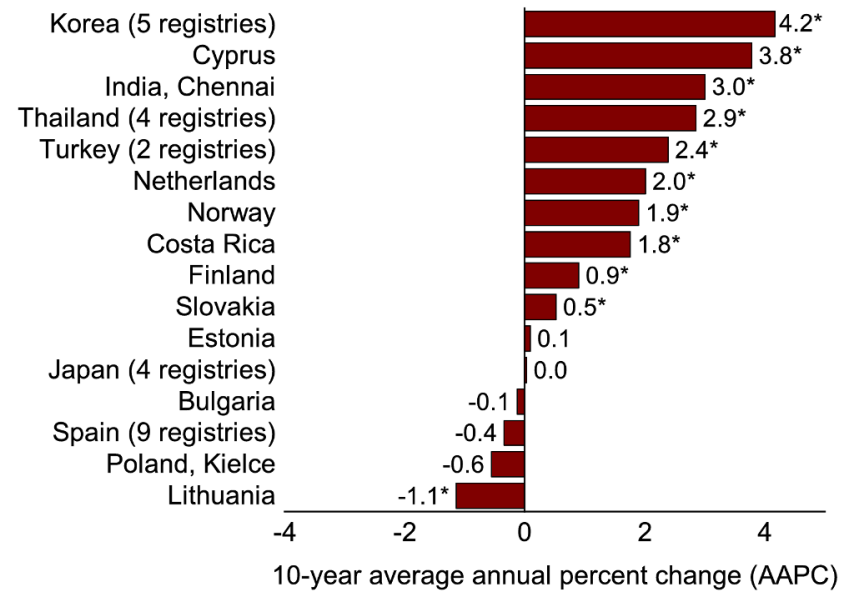

Ages $\geq \mathbf{5 0}$ years
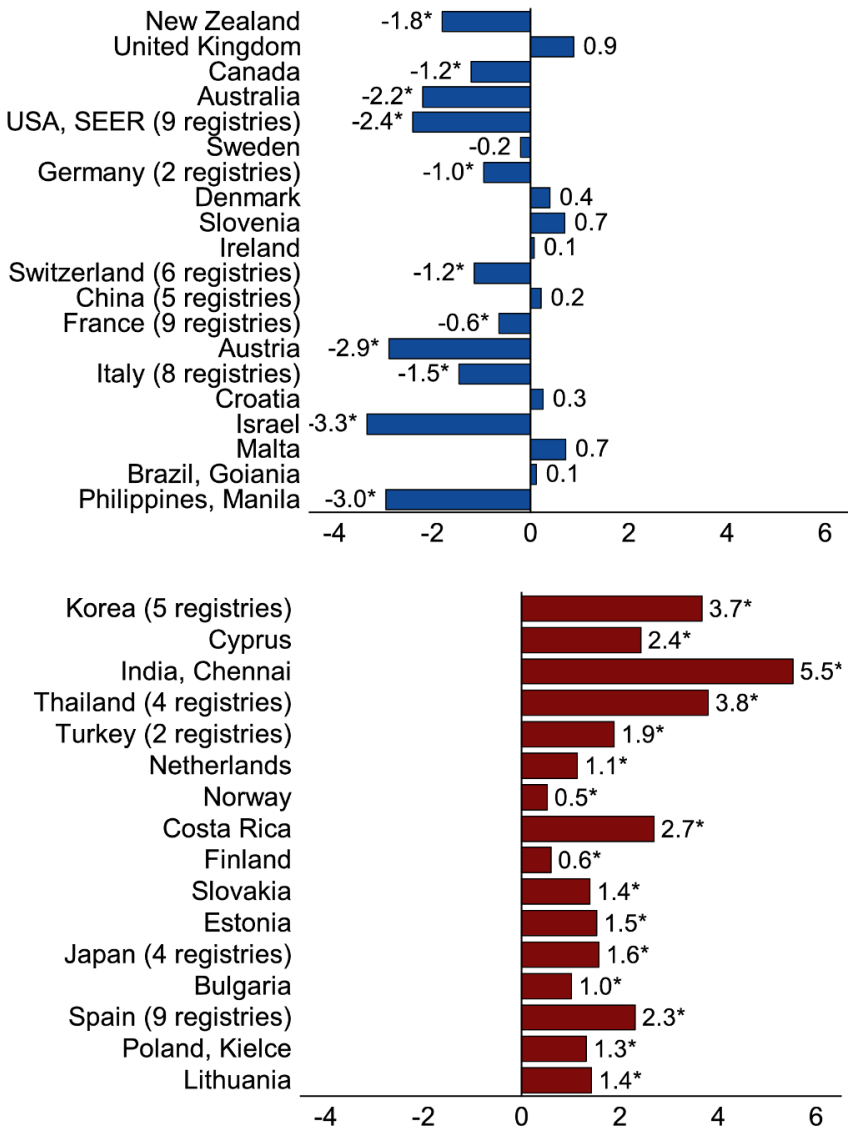

10 -year average annual percent change (AAPC)

Figure 2 Average annual per cent change (AAPC) in colorectal cancer incidence by age during the most recent 10 years of available data (A) countries with stable or declining trend among adults age 50 and older (B) countries with increasing trend among adults age 50 and older. AAPC reflects incidence during 2003-2012 except for Australia (2006-2015); Costa Rica (2002-2011); Finland (2007-2016); New Zealand (2007-2016); Norway (2008-2017); Slovakia (2001-2010); Sweden (2008-2017); USA (2007-2016). *AAPC is statistically significantly different from zero $(p<0.05)$ using a two-sided test based on the permutation method.

\section{CRC incidence trends}

Among 36 countries with a sufficient number of annual cases, CRC incidence in adults $<50$ during the past 10 years was stable in 14 countries and decreased in three-Austria, Italy and Lithuania-all by about $1 \%$ annually (online supplementary table 1 ). Incidence in adults 50 and older likewise declined in Austria and Italy, as well as in nine additional countries (online supplementary table 2). The increasing CRC trend in adults $<50$ in the remaining 19 countries was unique to that age group in nine countries (figure 2A). Among these nine countries, rates in older adults declined by $1 \%-2.4 \%$ per year in Germany, Canada, New Zealand, Australia, and USA, and were stable in UK, Sweden, Denmark, and Slovenia. Where data were available prior to 1990, the uptick in early-onset CRC began during 1992-1996 and was preceded by declining rates except in Slovenia, where there was a continuous increase of $0.8 \%$ per year from 1983 to 2012 (figure 3; online supplementary table 1).

Early-onset CRC incidence increased most rapidly in Korea (AAPC, 4.2 (95\% CI 3.4 to 5.0)), where rates rose at a similar pace among adults 50 and older (figure 2B). Incidence increased in both younger and older age groups in about one-quarter of countries examined; among these, the magnitude of the AAPC for young adults was notably larger than that for older adults in Cyprus, Netherlands and Norway. In Norway, for example, the
AAPC was 1.9 (95\% CI 1.4 to 2.5 ) among ages $20-49$ versus 0.5 (95\% CI 0.3 to 0.7 ) among ages 50 and older (figure $2 \mathrm{~B}$; online supplementary tables 1 and 2). In the Netherlands, the respective AAPCs were 2.0 (95\% CI 1.6 to 2.4 ) versus 1.1 (95\% CI 0.7 to 1.6), and the most recent linear (joinpoint) segment (2007-2012) was stable in older adults. Incidence in young adults began to increase in 1998 in Netherlands and 1996 in Norway according to joinpoint analysis, consistent with the timing of the trend in other high-income countries.

\section{Subsite-specific incidence trends}

Subsite-specific incidence trends varied with no clear pattern. For example, declines in early-onset CRC were confined to colon cancer in Italy and Lithuania, but to rectal cancer in Austria (online supplementary tables 3-4). In countries with increasing rates exclusively for early-onset disease, AAPCs were comparable for colon and rectal tumours in the USA, Sweden and Denmark; larger for, or confined to, colon tumours in Australia, New Zealand, Germany and UK; and larger for rectal tumours in Canada and Slovenia. Notably, rectal cancer incidence in the Netherlands increased among adults $<50$ years (AAPC, 1.9 (95\% CI 1.4 to 2.5$)$ ) but not among older adults (AAPC, $-0.1(95 \%$ CI -0.8 to 0.7$)$ ). Importantly, the interpretation of 


\section{A. North America and Oceania}
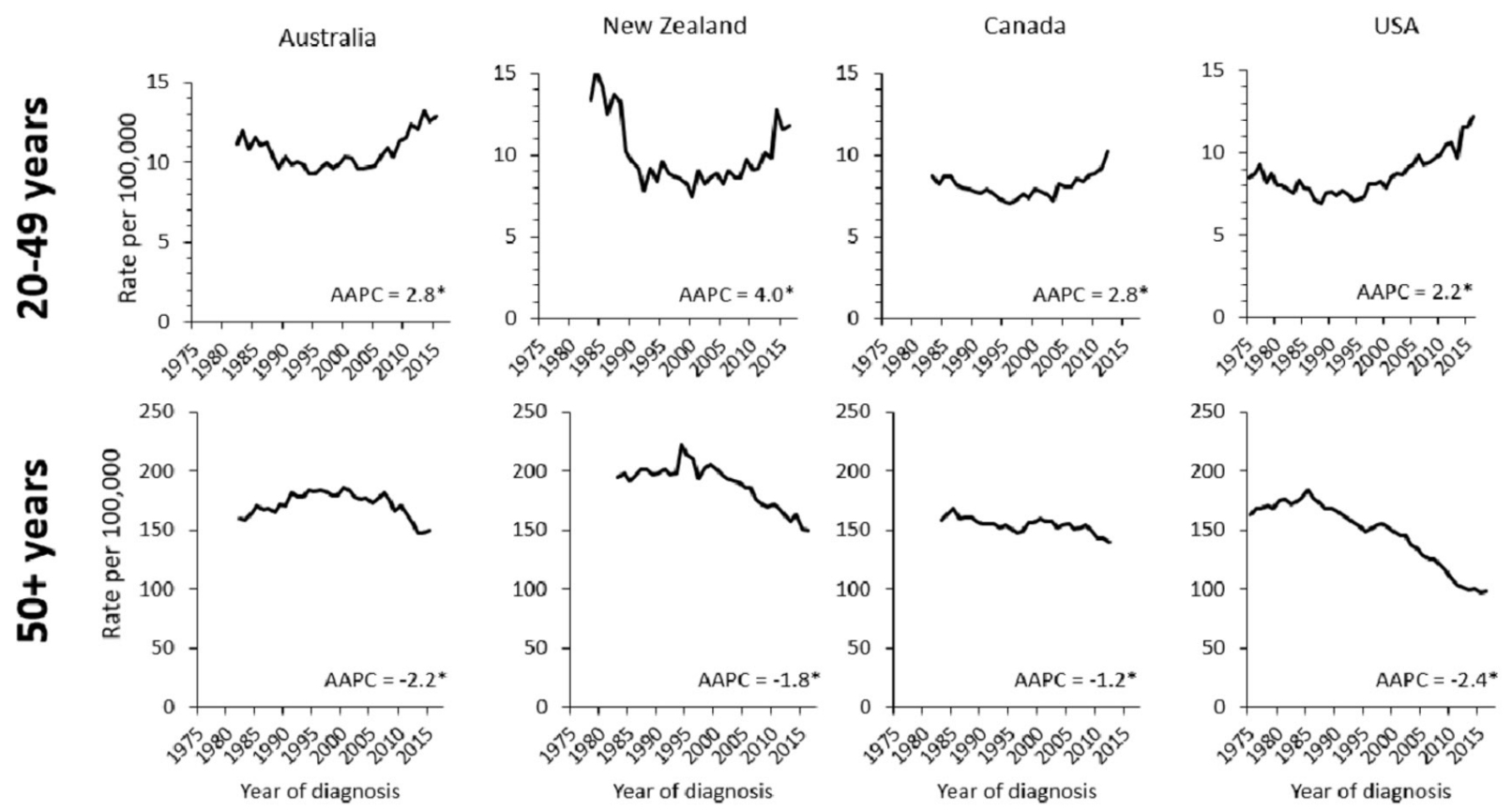

\section{B. Europe}
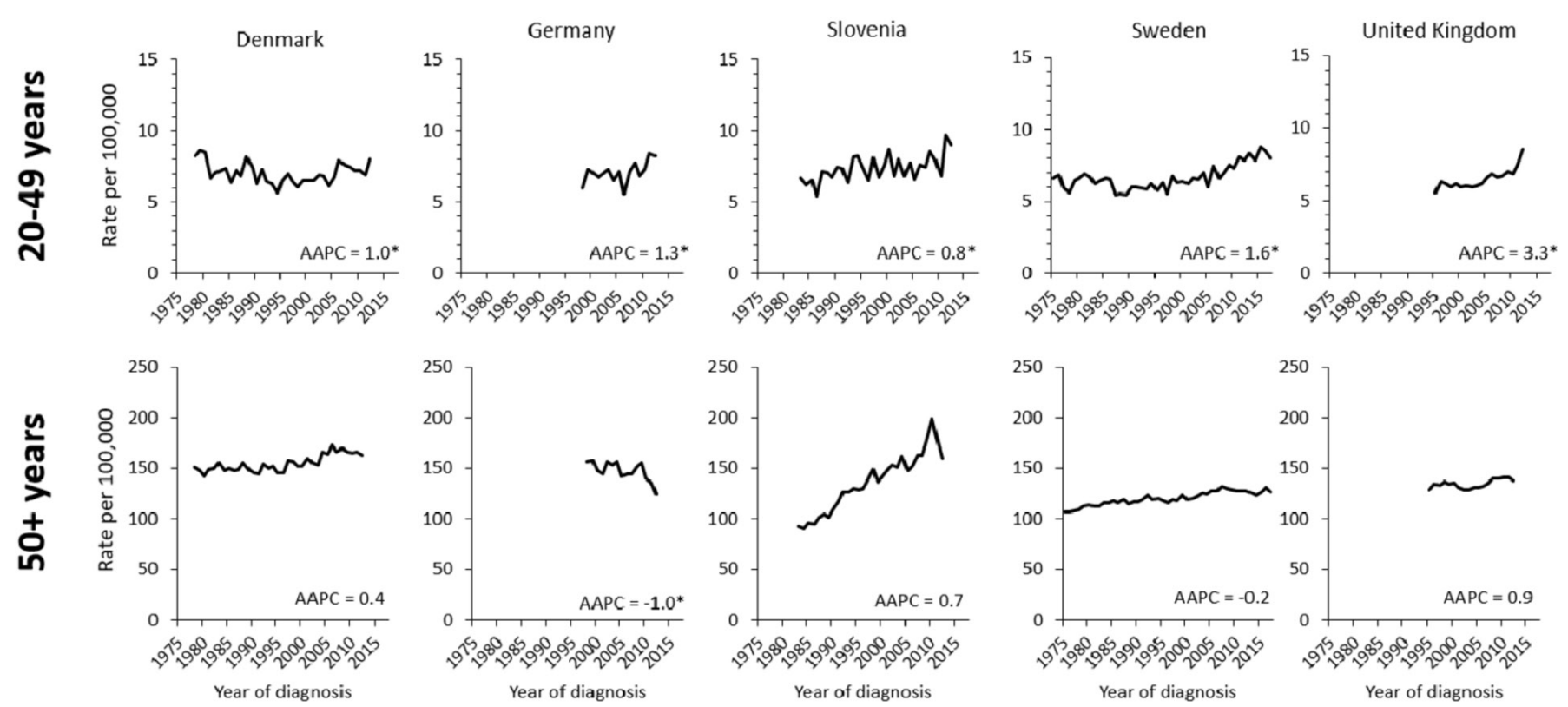

Figure 3 Colorectal cancer incidence trends by age, including the average annual per cent change (AAPC) during the most recent 10 years of available data, among countries with a unique increase in early-onset disease, by continent: (A) North America and Oceania (B) Europe. AAPC reflects incidence during 2003-2012 except for Australia (2006-2015); New Zealand (2007-2016); Sweden (2008-2017); USA (2007-2016). *AAPC is statistically significantly different from zero $(p<0.05)$ using a two-sided test based on the permutation method.

these subsite-specific differences is limited by the inclusion of appendiceal malignancies (C18.1) within the grouping for colon cancer (C18) in CI5 data. The AAPC for appendiceal cancer incidence in the USA during 2007-2016 was 15.5 (95\% CI 11.5 to 19.7) in ages $20-49$ years. ${ }^{19}$ We evaluated the extent to which the inclusion of appendiceal malignancies influenced our results by calculating AAPCs for CRC and colon cancer in the absence of appendiceal cancer for three countries (USA, New Zealand and Canada) for which these data were available. In the USA, the AAPC during 2007-2016 in ages 20-49 years excluding appendix was 1.7 (95\% CI 1.5 to 2.0 ) for CRC (vs 2.2 (95\% CI 1.9 to 2.5 ) including appendix) and 1.3 (95\% CI 1.0 to 1.7$)$ for 
colon cancer (vs 2.1 (95\% CI 1.7 to 2.6 ) including appendix) (online supplementary table 7). Thus, the AAPC for colon cancer (excluding appendix) is substantially smaller than that for rectal cancer $(2.1,95 \%$ CI 1.7 to 2.5$)$ whereas it previously appeared identical. Results were similar for New Zealand and Canada.

\section{DISCUSSION}

We found that the geographic variation in CRC incidence among adults ages 20-49 mirrors that in older adults, with a threefold difference between the highest (12.9 per 100000 in Korea) and lowest (3.5 per 100000 in India) rates. In contrast, age-specific temporal trends were variable, with a decline limited to three countries (Austria, Italy and Lithuania) for young-onset CRC versus 11 for older adults. Conversely, increasing incidence was unique to young adults in nine high-income countries (Australia, Canada, Denmark, Germany, New Zealand, Slovenia, Sweden, UK and USA) spanning three continents, often against a backdrop of rapidly declining rates in older adults. Similarly, increasing trends in Cyprus, Netherlands and Norway were twice as steep in young adults as in older adults. Our findings are consistent with previous, mostly country-level studies of age-related differences in temporal trends of CRC. ${ }^{5-9} 11$

The most rapid increases in early-onset CRC occurred in countries where rates are already highest, such as Korea, which had the same pattern for older adults. Reasons for the high and escalating burden in Korea are unclear, but may be related to the rapid dietary transition that took place in the wake of remarkable economic growth following the Korean war. ${ }^{20}$ Changes in the food supply were also initiated by the importation of wheat from the USA in response to food shortages in the late 1960s, which prompted the production of many wheat-derived processed foods during the 1970s. Shortly thereafter was the introduction of fast-food restaurants especially popular among youth. The obesity epidemic may also be a factor, given that East Asia has experienced among the largest relative increases in body mass index (BMI) worldwide among both adults and children. ${ }^{21}$ Asians have disproportionately high levels of visceral adiposity compared with Caucasians, ${ }^{22} 23$ which may more strongly influence CRC risk than BMI or waist circumference. ${ }^{24} 25$ Another potential contributor is the high prevalence of early-life antibiotic use, which has been associated with increased risk of colorectal adenoma, especially in the rectum. ${ }^{26} \mathrm{~A}$ recent study found that among six high-income countries, South Korea had the highest rate of paediatric antibiotic consumption, sevenfold higher than that in Norway, which had the lowest rate. ${ }^{27}$ The adenoma detection rate among Korean individuals in their 40s has been reported at almost $30 \%,{ }^{28}$ three times higher than that in Australians. ${ }^{29}$

Early-onset CRC also increased rapidly in countries where risk in older adults is declining at a similar pace, such as New Zealand, Australia, Canada and the USA. Reductions in CRC incidence among older adults in some countries are partly attributed to changing patterns in risk factors, such as reductions in smoking and widespread use of anti-inflammatory drugs. ${ }^{2} 30$ Likewise, rising incidence confined to young age groups signals changes in early life exposures that adversely influence CRC risk, particularly given the strong birth cohort effect apparent in the trend. ${ }^{511}$ Notably, eight of the nine countries with a unique rise in early-onset CRC had declining rates prior to the uptick beginning in the mid-1990s. Reductions in the prevalence of protective factors, such as physical activity (for colon cancer) and sufficient intake of dietary fibre, dairy, and fruits and vegetables, may play a role, as well as increased prevalence of obesity, smoking, red and/or process meat consumption, and/or excess alcohol consumption. $^{31}$

Obesity was recently found to be associated with a $20 \%$ excess risk of early-onset CRC, ${ }^{32}$ and prevalence has risen most rapidly in young adults ${ }^{33}$ and in English-speaking high-income countries, ${ }^{34}$ consistent with early-onset CRC patterns. However, increases in BMI are quite similar across Europe, despite varying CRC trends. For example, BMI increased from $24 \mathrm{~kg} / \mathrm{m}^{2}$ in 1975 to 27 in 2014 in men in Germany, where early-onset CRC increased, as well as in Austria, Croatia, Israel and Italy, where it did not. ${ }^{34} \mathrm{BMI}$ increases in women were smaller but also comparable. In addition, there are puzzling variations in the CRC trend by subsite, ${ }^{511}$ as well as by race/ethnicity and state within the USA, ${ }^{35}$ that suggest a role for risk factors beyond obesity. For example, obesity and a sedentary lifestyle are more strongly associated with colon tumours, ${ }^{37-40}$ yet rectal tumours appear to be driving the increase based on our findings herein and those of other studies. ${ }^{591135}$ If this is true, the rectal epithelium may be more exposed and/or susceptible to the carcinogenic mechanisms causing the increase in disease.

CRC risk is intrinsically linked to diet and its influence on gut immune response and inflammation. ${ }^{41}$ The global food supply has changed substantially in recent decades ${ }^{42}$ and evolving research is exploring the carcinogenic potential of relatively new food components. For example, associations have been uncovered between CRC and moderate consumption of sugarsweetened beverages, ${ }^{43}$ as well as high fructose corn syrup specifically in animal studies, ${ }^{44}$ both in the absence of obesity and metabolic syndrome. An inflammatory diet, characterised by high consumption of processed foods and high-glycaemic load carbohydrates, creates an environment conducive to colonic proliferation $^{45}$ and appears to increase CRC risk. ${ }^{46}$ What remains uncertain is how these dietary elements might influence early-life gut health. Importantly, the association between CRC and currently established risk factors is based almost entirely on disease occurrence in older aged cohorts. ${ }^{47}$

CRC screening programmes have mostly emerged over the past two decades and likely contributed to the declines in incidence among older adults in 11 countries. Of the 36 countries in our trend analysis, only four (Costa Rica, Cyprus, India and Philippines) lack a screening programme according to a recent global overview. ${ }^{48}$ In most countries, screening for CRC (generally with a stool test) is recommended to begin between the ages of 50 and 60 years. Exceptions are Italy, where screening begins at age 44 years, and China, Japan and Austria, where it begins at age 40. Notably, two (Austria and Italy) of the three countries where early-onset CRC declined have screened individuals beginning in their fourth decade since the early 1980 s. $^{48}$ Moreover, the decreasing trend among young adults in both Austria and Italy was confined to ages $40-49$ years, with rates in ages 20-39 years increasing by 3\% per year in Austria (data not shown). Although some of the rapid increases in early-onset CRC are reminiscent of the Korean thyroid cancer 'epidemic' that resulted from widespread ultrasound screening, ${ }^{49}$ overdetection of early-onset CRC is unlikely because screening before age 50 is rare in most countries, and mortality rates have also begun to rise. ${ }^{1250}$ After an extensive evidence review, the American Cancer Society recently lowered the recommended age to begin screening from 50 to 45 because disease risk in individuals under 50 has shifted substantially ${ }^{51}$ and Cancer Intervention and Surveillance Modeling Network modelling studies found a greater benefit to burden ratio for initiation at age 45 compared with $50 .{ }^{52-54}$ The US Preventive Services Task Force, the other US entity that issues cancer screening guidelines, is currently in 
the process of conducting an evidence review and expected to issue an updated recommendation by 2020-2021.

Our study is the first to provide a comprehensive global assessment of contemporary trends in early-onset CRC based on high-quality population-based cancer incidence information. However, the interpretation of subsite-specific differences in CI5 data is limited by the inclusion of appendiceal malignancies within the grouping for colon cancer. Accumulating evidence suggests that these tumours differ from those that develop in the colon in their biology and other characteristics. ${ }^{55}$ Inclusion of appendix attenuates comparisons of the burden for colon versus rectal cancer. Additionally, although appendiceal cancer accounts for only about $10 \%$ of cases in ages $<50$ years (data for USA, New Zealand and Canada), incidence rates are rising rapidly in high-income countries ${ }^{567}$ due to changes in classification and improved detection. This likely hindered our ability to detect the steeper rise for rectal than for colon cancer that has been reported by numerous studies of early-onset CRC trends excluding appendix, ${ }^{5911}$ but less often by those including appendix. ${ }^{12}$ Similarly, results from our sensitivity analyses that excluded appendix found larger increases for rectal than for colon tumours in each of the three countries with these data available. Incidence trends may also be influenced by temporal improvements in the quality of data, case capture, and specificity of coding. Other study limitations include incomplete population coverage for long-term incidence data in many countries; lack of more contemporary data (eg, since 2012) for most countries; and the absence of high-quality cancer registry data for the majority of low-income and middle-income countries.

In summary, CRC incidence rates uniquely increased in young adults over the past two decades in nine high-income countries spanning three continents, often in sharp contrast to rapid declines in older adults. These patterns potentially signal changes in early-age exposures conducive to large bowel carcinogenesis and highlight an urgent need for research to explore the potentially unique aetiology of young-onset CRC. Beyond awaiting scientific discovery, clinicians have an opportunity to help mitigate premature morbidity and mortality from CRC with active documentation of familial cancer history; timely follow-up of symptoms, regardless of patient age; and screening when appropriate.

Acknowledgements The authors gratefully acknowledge all cancer registries and their staff for their hard work and diligence in collecting cancer information, without which this research could not have been done.

Contributors Study concept and design: RS, AJ. Analysis and interpretation of the data: RS, LT, AJ, IS. Drafting the manuscript: RS, LAT. Critical revision of the manuscript for important intellectual content: all authors.

Funding The authors have not declared a specific grant for this research from any funding agency in the public, commercial or not-for-profit sectors.

Competing interests None declared.

Patient consent for publication Not required.

Provenance and peer review Not commissioned; externally peer reviewed.

Data availability statement Data are available in a public, open access repository. Data are available upon reasonable request. All data relevant to the study are included in the article or uploaded as supplementary information.

\section{ORCID iD}

Rebecca L Siegel http://orcid.org/0000-0001-5247-8522

\section{REFERENCES}

1 Bray F, Ferlay J, Soerjomataram I, et al. Global cancer statistics 2018: GLOBOCAN estimates of incidence and mortality worldwide for 36 cancers in 185 countries. CA Cancer J Clin 2018;68:394-424.

2 Arnold M, Sierra MS, Laversanne M, et al. Global patterns and trends in colorectal cancer incidence and mortality. Gut 2017;66:683-91.
3 Austin $\mathrm{H}$, Henley SJ, King J, et al. Changes in colorectal cancer incidence rates in young and older adults in the United States: what does it tell us about screening. Cancer Causes Control 2014;25:191-201.

4 Bailey CE, Hu C-Y, You YN, et al. Increasing disparities in the age-related incidences of colon and rectal cancers in the United States, 1975-2010. JAMA Surg 2015;150:17-22.

5 Siegel RL, Fedewa SA, Anderson WF, et al. Colorectal cancer incidence patterns in the United States, 1974-2013. J Nat/ Cancer Inst 2017;109:djw322.

6 Siegel RL, Jemal A, Ward EM. Increase in incidence of colorectal cancer among young men and women in the United States. Cancer Epidemiol Biomark Prev 2009;18:1695-8.

7 Young JP, Win AK, Rosty C, et al. Rising incidence of early-onset colorectal cancer in Australia over two decades: report and review. J Gastroenterol Hepatol 2015;30:6-13

8 Feletto E, Yu XQ, Lew J-B, et al. Trends in colon and rectal cancer incidence in Australia from 1982 to 2014: analysis of data on over 375,000 cases. Cancer Epidemiol Biomarkers Prev 2019:28:83-90.

9 Brenner DR, Ruan Y, Shaw E, et al. Increasing colorectal cancer incidence trends among younger adults in Canada. Prev Med 2017;105:345-9.

10 Doll R. Progress against cancer: an epidemiologic assessment. The 1991 John C. Cassel memorial lecture. Am J Epidemiol 1991;134:675-88.

11 Araghi M, Soerjomataram I, Bardot A, et al. Changes in colorectal cancer incidence in seven high-income countries: a population-based study. Lancet Gastroenterol Hepatol 2019:4:511-8

12 Vuik FER, Nieuwenburg SAV, Bardou M, et al. Increasing incidence of colorectal cancer in young adults in Europe over the last 25 years. Gut 2019;68:1820-6.

13 Lui RN, Tsoi KKF, Ho JMW, et al. Global increasing incidence of young-onset colorectal cancer across 5 continents: a joinpoint regression analysis of 1,922,167 cases. Cancer Epidemiol Biomarkers Prev 2019;28:1275-82.

14 Ferlay J, Colombet M, Bray F. Cancer Incidence in Five Continents, CI5plus: IARC CancerBase No. 9. Lyon, France: International Agency for Research on Cancer, 2018.

15 Patel P, De P. Trends in colorectal cancer incidence and related lifestyle risk factors in 15-49-year-olds in Canada, 1969-2010. Cancer Epidemiol 2016:42:90-100.

16 Troeung L, Sodhi-Berry N, Martini A, et al. Increasing incidence of colorectal cancer in adolescents and young adults aged 15-39 years in Western Australia 1982-2007: examination of colonoscopy history. Front Public Health 2017;5.

$17 \mathrm{Kim} \mathrm{H-J,} \mathrm{Fay} \mathrm{MP,} \mathrm{Feuer} \mathrm{EJ,} \mathrm{et} \mathrm{al.} \mathrm{Permutation} \mathrm{tests} \mathrm{for} \mathrm{joinpoint} \mathrm{regression} \mathrm{with}$ applications to cancer rates. Stat Med 2000;19:335-51.

18 Doll R, Cook P. Summarizing indices for comparison of cancer incidence data. Int Cancer 1967:2:269-79.

19 Surveillance, Epidemiology and End Results Program. SEER *Stat Database: Incidence - SEER 9 Regs Research Data with Delay-Adjustment, Malignant Only, (1975-2016)<Katrina/Rita Population Adjustment>National Cancer Institute, DCCPS, Surveillance Research Program, Surveillance Systems Branch, released April 2019 based on the November 2018 submission 2019.

20 Kim S, Moon S, Popkin BM. The nutrition transition in South Korea. Am J Clin Nutr 2000;71:44-53.

21 Sung $H$, Siegel RL, Torre LA, et al. Global patterns in excess body weight and the associated cancer burden. CA Cancer J Clin 2019;69:88-112.

22 Kadowaki T, Sekikawa A, Murata K, et al. Japanese men have larger areas of visceral adipose tissue than Caucasian men in the same levels of waist circumference in a population-based study. Int J Obes 2006;30:1163-5.

23 Park Y-W, Allison DB, Heymsfield SB, et al. Larger amounts of visceral adipose tissue in Asian Americans. Obes Res 2001;9:381-7

24 Keum N, Lee DH, Kim R, et al. Visceral adiposity and colorectal adenomas: doseresponse meta-analysis of observational studies. Ann Oncol 2015;26:1101-9.

25 Nam SY, Kim BC, Han KS, et al. Abdominal visceral adipose tissue predicts risk of colorectal adenoma in both sexes. Clin Gastroenterol Hepatol 2010;8:443-50.

26 Cao Y, Wu K, Mehta R, et al. Long-Term use of antibiotics and risk of colorectal adenoma. Gut 2018;67:672-8.

27 Youngster I, Avorn J, Belleudi V, et al. Antibiotic use in children - a cross-national analysis of 6 countries. J Pediatr 2017;182:239-44.

28 Bae T, Ha Y, Kim C, et al. Distribution of the colonoscopic adenoma detection rate according to age: is recommending colonoscopy screening for Koreans over the age of 50 safe? Ann Coloproctol 2015;31:46-51.

29 Wong S, Lidums I, Rosty C, et al. Findings in young adults at colonoscopy from a hospital service database audit. BMC Gastroenterol 2017;17:56.

30 Edwards BK, Ward E, Kohler BA, et al. Annual report to the nation on the status of cancer, 1975-2006, featuring colorectal cancer trends and impact of interventions (risk factors, screening, and treatment) to reduce future rates. Cancer 2010;116:544-73.

31 Gunter MJ, Alhomoud S, Arnold M, et al. Meeting report from the joint IARC-NCI International cancer seminar series: a focus on colorectal cancer. Ann Oncol 2019:30:510-9.

32 Liu P-H, Wu K, Ng K, et al. Association of obesity with risk of early-onset colorectal cancer among women. JAMA Oncol 2019;5:37-44

33 Afshin A, Forouzanfar MH, Reitsma MB, et al. Health effects of overweight and obesity in 195 countries over 25 years. N Engl J Med 2017;377:13-27. 
34 Collaboration NCDRF. Trends in adult body-mass index in 200 countries from 1975 to 2014: a pooled analysis of 1698 population-based measurement studies with $19 \cdot 2$ million participants. The Lancet 2016;387:1377-96.

35 Siegel RL, Medhanie GA, Fedewa SA, et al. State variation in early-onset colorectal cancer in the United States, 1995-2015. J Nat/ Cancer Inst 2019;25.

36 Araghi M, Fidler MM, Arnold M, et al. The future burden of colorectal cancer among US blacks and whites. J Natl Cancer Inst 2018;110:791-3.

37 Boyle T, Keegel T, Bull F, et al. Physical activity and risks of proximal and distal colon cancers: a systematic review and meta-analysis. J Nat/ Cancer Inst 2012;104:1548-61.

38 Ma Y, Yang Y, Wang F, et al. Obesity and risk of colorectal cancer: a systematic review of prospective studies. PLoS One 2013;8:e53916.

39 Moghaddam AA, Woodward M, Huxley R. Obesity and risk of colorectal cancer: a meta-analysis of 31 studies with 70,000 events. Cancer Epidemiol Biomark Prev 2007;16:2533-47.

40 Robsahm TE, Aagnes B, Hjartaker A, et al. Body mass index, physical activity, and colorectal cancer by anatomical subsites: a systematic review and meta-analysis of cohort studies. Eur J Cancer Prev 2013;22:492-505.

41 O'Keefe SJD, Diet O'Keefe SJ.. Diet, microorganisms and their metabolites, and colon cancer. Nat Rev Gastroenterol Hepatol 2016;13:691-706.

42 Schmidhuber J, Sur P, Fay K, et al. The global nutrient database: availability of macronutrients and micronutrients in 195 countries from 1980 to 2013. The Lancet Planetary Health 2018;2:e353-68.

43 Fuchs MA, Sato K, Niedzwiecki D, et al. Sugar-Sweetened beverage intake and cancer recurrence and survival in CALGB 89803 (Alliance). PLoS One 2014;9:e99816.

44 Goncalves MD, Lu C, Tutnauer J, et al. High-Fructose corn syrup enhances intestinal tumor growth in mice. Science 2019;363:1345-9.

45 O'Keefe SJD, Li JV, Lahti L, et al. Fat, fibre and cancer risk in African Americans and rural Africans. Nat Commun 2015;6:6342.
46 Tabung FK, Liu L, Wang W, et al. Association of dietary inflammatory potential with colorectal cancer risk in men and women. JAMA Oncol 2018;4:366-73.

47 Nimptsch K, Wu K. Is timing important? the role of diet and lifestyle during early life on colorectal neoplasia. Curr Colorectal Cancer Rep 2018;14:1-11.

48 Schreuders EH, Ruco A, Rabeneck L, et al. Colorectal cancer screening: a global overview of existing programmes. Gut 2015;64:1637-49.

49 Park S, Oh C-M, Cho H, et al. Association between screening and the thyroid cancer "epidemic" in South Korea: evidence from a nationwide study. BMJ 2016;355.

50 Siegel RL, Miller KD, Jemal A. Colorectal cancer mortality rates in adults aged 20 to 54 years in the United States, 1970-2014. JAMA 2017;318:572-4.

51 Anderson JC, Samadder JN. To screen or not to screen adults $45-49$ years of age: that is the question. Am J Gastroenterol 2018;113:1750-3.

52 Knudsen $A B$, Zauber $A G$, Rutter $C M$, et al. Estimation of benefits, burden, and harms of colorectal cancer screening strategies: modeling study for the US preventive services Task force. JAMA 2016;315.

53 Wolf AMD, Fontham ETH, Church TR, et al. Colorectal cancer screening for averagerisk adults: 2018 guideline update from the American cancer Society. CA Cancer J Clin 2018;68:250-81.

54 Peterse EFP, Meester RGS, Siegel RL, et al. The impact of the rising colorectal cancer incidence in young adults on the optimal age to start screening: Microsimulation analysis I to inform the American cancer Society colorectal cancer screening guideline. Cancer 2018;124:2964-73.

55 CS-P A, Shen JP, Hardy-Abeloos CJ, et al. Genomic landscape of appendiceal neoplasms. JCO Precision Oncology 2018:1-18.

56 Marmor S, Portschy PR, Tuttle TM, et al. The rise in appendiceal cancer incidence: 2000-2009. J Gastrointest Surg 2015;19:743-50.

57 van den Heuvel MGW, Lemmens VEPP, Verhoeven RHA, et al. The incidence of mucinous appendiceal malignancies: a population-based study. Int J Colorectal Dis 2013;28:1307-10. 